\title{
评述
}

\section{MALDI-TOF MS技术在传染病诊断中的应用进展}

\author{
王立琴 ${ }^{1,2}$, 张驰 ${ }^{1,2}$, 李晓东 ${ }^{3}$, 彭俊平 $1,2^{*}$ \\ 1. 中国医学科学院\&北京协和医学院病原生物学研究所, 国家卫生健康委病原系统生物学重点实验室, 北京 100176; \\ 2. 中国医学科学院呼吸道疾病病原组研究重点实验室, 北京 100176 ; \\ 3. 岛津企业管理(中国)有限公司, 北京 100020 \\ *联系人, E-mail: pengjp@ipbcams.ac.cn
}

收稿日期: 2021-08-23; 接受日期: 2021-10-21; 网络版发表日期: 2022-03-30

中国医学科学院中央级公益性科研院所基本科研业务费(批准号: 2019PT310029)资助

摘要 基质辅助激光解吸电离飞行时间质谱(matrix-assisted laser desorption/ionization time-of-flight mass spectrometry, MALDI-TOF MS)作为新兴的检测手段已被广泛应用于临床微生物实验室. 相比传统基于表型的检 测方法, 如形态学、血清学及生化检测, MALDI-TOF MS 可在短时间内对待测微生物进行物种鉴定及耐药表型分 析, 简化了操作流程. 多项研究表明, MALDI-TOF MS在病原生物学领域具备较大的应用潜能, 通过分析分子或离 子的质荷比以识别或量化待测物，适用于多种类别分子的检测. 依据检测靶标的不同，本文将从蛋白质与核酸两 方面系统综述该技术在传染病诊断中的应用进展.

关键词 MALDI-TOF MS, 菌种鉴定, 耐药性检测, 病原体检测与分型

病原生物学是当前生命科学研究的前沿领域，对 病原体进行快速准确的鉴定是传染病诊断中的关键环 节. 近年来, 随着检测方法日趋完善与发展, 病原体鉴 定与分型工作逐渐体系化．传统的鉴定方法主要基于 表型分析，包括形态学鉴定、生化鉴定和血清学鉴定 等 ${ }^{[1]}$. 传统病原体分离鉴定手段作为病原体鉴定的金 标准，其检测结果直观，且获取纯种菌株后方便开展 后续鉴定及耐药分析工作, 但通常实验过程耗时较长, 接种需求量较大, 结果判定受制于多步骤综合分析, 难 以满足微生物快速鉴定的需求. 因此, 分子生物学技术 应运而生，聚合酶链式反应、荧光原位杂交及基因测 序等技术的发展为传染病诊断工作提供了强大助力.
同时, 基质辅助激光解吸电离飞行时间质谱(matrix-assisted laser desorption/ionization time-of-flight mass spectrometry，MALDI-TOF MS)作为一种高效简便的 分子检测方法，开启了质谱技术应用于生物大分子检 测的先河. 日本科学家田中耕一与美国科学家John B. Fenn也因基于 “软性解吸附电离分析”的MALDI-TOF MS技术被授予2002年诺贝尔化学奖. MALDI-TOF MS技术将表型分析与分子分析结合起来, 目前已广泛 应用于微生物鉴定分型与抗菌药物耐药性检测. 尽管 质谱设备购置的最初成本较高, 但单个样本分析的成 本低于传统基于表型的方法 ${ }^{[2]}$, 且极大程度上缩短了 分析过程的周转时间 ${ }^{[3]}$, 为传染病的快速诊断与临床

引用格式: 王立琴, 张驰, 李晓东, 等. MALDI-TOF MS技术在传染病诊断中的应用进展. 中国科学: 生命科学, 2022, 52: 1858-1867

Wang L Q, Zhang C, Li X D, et al. Progress in applications of MALDI-TOF MS technology in the diagnosis of infectious diseases (in Chinese). Sci Sin Vitae, 2022, 52: 1858-1867, doi: 10.1360/SSV-2021-0317 
决策提供依据. 伴随硬件设备的性能提升与软件数据 库的完善扩充，该技术现已在临床微生物实验室的病 原体鉴定与分型及抗生素耐药性检测方面发挥重要作 用. 本文将分别从蛋白质检测与核酸检测的层面, 系统 阐述MALDI-TOF MS在传染病诊断方面的应用进展.

\section{MALDI-TOF MS技术概述}

MALDI-TOF MS主要由两部分组成: 基质辅助激 光解吸电离离子源(MALDI) 和飞行时间质量分析器 (TOF). 离子源通过激光轰击待测样品与基质形成的共 结晶薄膜, 使基质从中吸收能量并传递给生物分子, 两 者间发生质子转移同时伴随生物分子电离，电离后的 生物分子在电场作用下加速通过飞行管道，依据待测 离子的质荷比 $(\mathrm{m} / \mathrm{z})$ 与飞行时间成正比的关系，对不同 飞行时间的离子进行差异化质量分析 ${ }^{[4]}$. 其中, MALDI 作为一种软电离技术, 使得待测分子在离子化过程中 产生较少的碎片离子, 质谱图中不同大小的离子呈有 序排布，便于对谱图进行分析，与同样基于软电离方 法的电喷雾电离质谱(electrospray ionization mass spectrometry, ESI-MS)相比, 主要区别在于样品的离子化方 式不同. 电喷雾电离利用高电压与雾化气的作用, 使待
测样本溶液在电喷雾针端出口处形成细小的带电液 滴, 伴随溶剂挥发与液滴变小, 表面电荷排斥力加强, 最终离子由溶剂相转移至气相, 适用于极性化合物及 离子化合物的分析; MALDI利用基质转移脉冲式激光 的能量, 使得热敏或不易挥发的分析物由固相直接转 化为离子, 同时避免过度电离为碎片离子, 产生的离 子通常只带一个电荷, 适用于生物大分子, 如肽类、 核酸类化合物的检测 ${ }^{[5]}$.

\section{MALDI-TOF MS技术在蛋白质检测方 面的应用}

作为软电离技术手段之一, MALDI-TOF MS适用 于蛋白质组学研究中各类生物大分子的检测. 鉴于其 直接反馈待测分子的质量变化, 而非依赖苂光、酶-底 物等间接反应, 应用于传染病诊断领域时, 在菌种鉴定 与而药性检测方面均表现出良好的适用性与实效性 (图1).

\section{1 菌种鉴定}

蛋白质的下游产物肽经质谱分析生成肽质量指纹 图谱(peptide mass fingerprinting, PMF) ${ }^{[6]}$, 每一种细菌

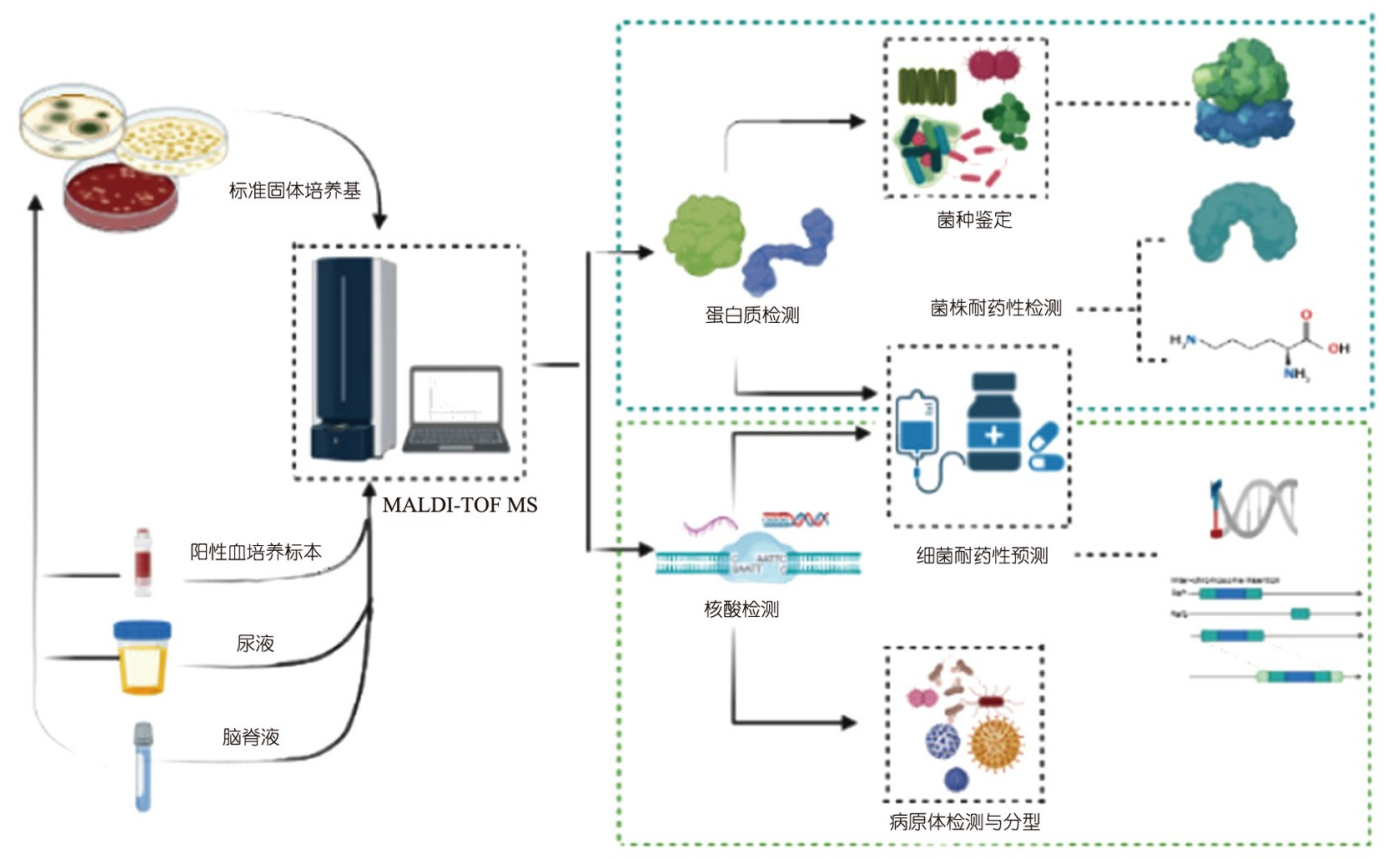

图 1 MALDI-TOF MS在蛋白质检测及核酸检测方面的应用

Figure 1 Application of MALDI-TOF MS in protein detection and nucleic acid detection 
都具备其特征性的PMF，且主要由核糖体蛋白组成 ${ }^{[7]}$. 通过MALDI-TOF MS获得目标细菌高丰度稳定表达 的指纹图谱，与数据库中已有图谱进行比对分析，从 而实现菌株在属、种水平上的快速鉴定(表1).

（1）标准固体培养基来源的菌种鉴定. 经典的 MALDI-TOF MS分析流程, 是取琼脂平板上生长状况 典型的单个菌落, 点样于靶板上, 待其自然干燥后与基 质混合形成共结晶膜，经电离后进行数据采集与结果 鉴定，目前已在葡萄球菌、大肠杆菌、肺炎克雷伯 菌、沙门氏菌、志贺氏菌、铜绿假单胞菌等不同菌株 的鉴定中得到广泛应用 ${ }^{[8]}$. 而某些细菌的内部蛋白被 多糖或包膜包裹, 如分枝杆菌和部分酵母菌 ${ }^{[9]}$, 单纯通 过上述处理难以获取其内部蛋白. 因此, 在上样分析前 需进行一定的样品前处理, 乙醇-甲酸萃取法 ${ }^{[10]}$ 作为常 规的样品前处理手段, 可实现大部分微生物的有效鉴 定. O'Connor等人 ${ }^{[11]}$ 设计二氧化硅/氧化锆珠结合超声 振动两步法实现结核分枝杆菌复合体(Mycobacterium tuberculosis complex, MTC)细胞壁的物理破坏，而后使 用乙醇、甲酸和乙腈提取结核分枝杆菌复合体蛋白, 结果表明，与布鲁克分枝杆菌提取方法(MycoEX)相 比，该方法鉴定水平显著提升(两步法: 属水平鉴定准 确率达 $100 \%$ 、种水平鉴定准确率达 $85 \%$; MycoEX: 属 水平鉴定准确率达 $79 \%$ 、种水平鉴定准确率达 $62 \%$ ).

(2) 阳性血培养标本与尿液、脑脊液等临床样本
的菌种鉴定．对于病程发展迅速、临床症状严重的细 菌感染性疾病，常规标准的菌种培养方法无法满足快 速鉴定的需求，亟需建立针对临床标本或短时培养样 本的直接检测手段. MALDI-TOF MS是目前可直接用 于阳性血培养标本及尿液、脑脊液等临床样本中病原 体鉴定的优势技术之一 ${ }^{[12]}$ ，通过缩减菌种培养耗费的 时长, 为危重患者的诊断与治疗争取时间.

应用MALDI-TOF MS对血液培养物或尿液等临 床样本进行检测时，需考虑到血细胞或来自宿主与培 养基的蛋白质对分析的影响，因此，适当的样品预处 理是准确鉴定病原体的前提. 已有研究报道了不同处 理方法的应用效果，其中，Sepsityper®试剂盒广泛应 用于血源样品的前处理，通过混合裂解缓冲液与血液 培养物以分解样品中的血液成分, 随后通过高速离心 收集细菌 ${ }^{[13]}$; 尿液前处理基于逐步离心, 洗涤, 过滤和 蛋白质提取的方案 ${ }^{[14]}$ 成本相对较低，但标准化程度有 待提升，需要在进一步应用中优化出成本和效益两相 平衡的前处理方法; 为诊断肺炎克雷伯菌引发的细菌 性脑膜炎, 有研究针对脑脊液样本进行离心洗涤, 而 后利用乙醇-甲酸萃取法提取细菌内容物用于检测 ${ }^{[15]}$. 但应注意的是, 在实践应用中, 临床样本中较低的细菌 载量可能会限制这种方法的使用.

(3) 用于菌种鉴定的商用MALDI-TOF MS系统性 能比较. MALDI-TOF MS正逐步完善成为一种快速、

表 1 MALDI-TOF MS技术在传染病诊断方面的应用及依据

Table 1 The application and basis of MALDI-TOF MS in the diagnosis of infectious diseases

\begin{tabular}{|c|c|c|c|}
\hline 检测靶标 & 应用 & 依据 & 参考文献 \\
\hline \multirow[b]{2}{*}{ 蛋白质 } & 菌种鉴定 & $\begin{array}{c}\text { MALDI-TOF MS可对细菌相应肽或蛋白质进行解吸分析, 所得特征性的肽质量指纹 } \\
\text { 图谱与数据库中已有谱图进行比对, 构成对细菌进行分类鉴定的基础 }\end{array}$ & {$[6 \sim 21]$} \\
\hline & 菌株耐药性检测 & $\begin{array}{c}\text { 1. 针对可产生抗生素水解酶或针化酶的耐药菌, 应用MALDI-TOF MS检测抗生素水 } \\
\text { 解或修饰前后质谱峰的偏移, 以鉴别细菌的耐药性与敏感性 } \\
\text { 2. 针对非产酶机制的耐药菌, 可对细菌摄入的氨基酸进行同位素标记, 依据耐药菌株 } \\
\text { 与每感菌株代谢水平差异实现间接检测 } \\
\text { 3. 基于耐药菌株与敏感菌株蛋白质表达差异, 以MALDI-TOF MS检测特异性蛋白质 } \\
\text { 谱峰, 依据单峰或峰簇差异可进一步别敏感株与耐药株 }\end{array}$ & {$[22 \sim 29,31 \sim 35]$} \\
\hline \multirow[t]{2}{*}{ 核酸 } & 病原体检测与分型 & $\begin{array}{c}\text { MALDI-TOF MS可区分单碱基的质量差异, 以其应用于SNP检测为例, } \\
\text { 技术原理为: } \\
\text { 1. 利用多重PCR技术扩增包含SNP位点在内的核酸序列 } \\
\text { 2. 针对每个靶点SNP设计一条延伸引物, 在以ddNTPs为底物的体系中进 } \\
\text { 行单碱基延伸 } \\
\text { 3. 利用MALDI-TOF MS对延伸产物进行分析, 依据待测样品的核苷酸多态性对病原 } \\
\text { 体进行检测与分型 }\end{array}$ & {$[37,39 \sim 53]$} \\
\hline & 细菌耐药性预测 & $\begin{array}{c}\text { 基因自发突变或种内/种间遗传元件转移均有可能引发耐药性, 利用MALDI-TOF MS } \\
\text { 检测耐药相关基因可预测菌株耐药性 }\end{array}$ & {$[52,54,55]$} \\
\hline
\end{tabular}


准确且具备成本效益的方法, 目前已有多个商用系统 用于菌种鉴定. 本文选择三种常见的商用MALDITOF MS系统, 即BioTyper ${ }^{\circledR}$ (Bruker Daltonics, Germany)、VITEK ${ }^{\circledR}$ MS Plus(bioMérieux, 硬件为岛津 OEM产品)和Autof ms 1000(Autobio Diagnostics, Chi$\mathrm{na})^{[16]}$ 进行比较. 多项研究评估了三种系统应用于细菌 及真菌鉴定方面的性能. Park等人 ${ }^{[17]}$ 选取205个保存菌 株和302个临床分离株作为测试样本, 对Autof ms 1000 与Bruker Biotyper系统进行性能比较, 应用 $16 \mathrm{~S}$ rRNA 或ITS区序列分析对鉴定结果存在差异的菌株进行鉴 定. 研究表明, 保存菌株中, Autof ms 1000与Bruker Biotyper在物种水平上的鉴定准确率分别达 $98.5 \%$, $97.6 \%$; 在临床分离株中则分别为 $99.7 \%, 99.3 \%$; Lin等 人 $^{[18]}$ 比较了VITEK MS和Bruker Biotyper系统在鉴定 临床分离株时的性能, 发现两者对假单胞菌的准确鉴 定率分别为 $98.7 \%$ 与 $100 \%$. 上述研究提示, 三种系统 应用于常规临床微生物鉴定时, 相互之间具备可比性. 另外, 一些研究通过联合商用MALDI-TOF系统与其他 数据分析系统, 进一步优化鉴定功能, 例如, Wei等 人 ${ }^{[19]}$ 开发了一种Biotyper与ClinProTools双系统联合的 方法, 两者同为Bruker Daltonics公司产品, 安装于同一 台质谱仪中, Biotyper用于获取测试菌株的原始谱图, 基于ClinProTools系统构建的遗传算法(genetic algorithm, GA)生成分类模型, 以区分炭疽芽孢杆菌和蜡 样芽狍杆菌分离株, 特异性和准确性均达 $100 \%$, 方法 兼具时效性、高通量及生物安全要求.

针对罕见微生物鉴定的数据库完备程度是各系统 性能比较的关键所在. 目前多数评价不同MALDI-TOF MS系统的研究均基于临床实验室中常见的微生物, 少 有研究选择难以鉴定或罕见微生物作为测试样本. Lévesque等人 ${ }^{[20]}$ 同时纳入常规微生物与罕见微生物对 Bruker Biotyper与VITEK MS系统的性能进行比较, 发 现两种系统均能对包含革兰氏阳性细菌、革兰氏阴性 细菌、分枝杆菌等在内的常规微生物进行高准确性 (>85\%)的鉴定, 存异不大(Biotyper: 86.4\%; VITEK MS: $92.3 \%$ ), 但在鉴定放线菌和丝状真菌等罕见微生物时, 与Bruker Biotyper相比, VITEK MS容易给出错误结果. $\mathrm{Yi}$ 等人 ${ }^{[21]}$ 选取一千余株包含不同菌种复合体的酵母菌 样本系统评估了Autof ms 1000和Vitek MS应用于酵母 菌种鉴定的有效性, 以ITS测序作为参比方法, 结果显 示, Autof ms 1000和Vitek MS系统分别正确鉴定出
$99.2 \%$ 和 $89.2 \%$ 的分离物, 属水平错误识别率分别为 $0.4 \%$ 和 $1.6 \%$, 种水平错误识别率分别为 $0.1 \%$ 和 $3.5 \%$, 表明Autof ms 1000在酵母鉴定中表现出较好的能力, 而Vitek MS在该项应用中鉴定准确性不及前者, 尤其 当研究对象为系统发育树上密切相关的罕见物种时. 上述研究提示, 在临床实验室中应用单一检测平台进 行微生物鉴定时, 应当充分考虑不同质谱平台的性能 特点.

\section{2 基于蛋白质质谱的菌株耐药性检测}

不同程度的抗菌药物耐药性已经发展为日益严重 的公共卫生问题，对耐药性进行快速检测是制定有效 抗生素治疗方案的前提. MALDI-TOF MS除常规应用 于菌种鉴定外, 在耐药性检测方面的应用也逐渐深入. 目前基于该技术已经建立了多种以蛋白质为靶标的耐 药性检测方法, 例如, 细菌产酶机制诱导下抗生素降解 或修饰产物的质谱检测、稳定同位素(非放射性)标记 的氨基酸检测以及耐药相关生物标志物的特征性质谱 峰检测等 ${ }^{[22]}$ (表1).

(1) 细菌产酶机制诱导下抗生素降解或修饰产物 的质谱检测. 耐药相关酶如水解酶、钝化酶的产生为 建立抗菌药物治疗方案带来挑战. 其中以染色体或质 粒介导产生的 $\beta$-内酰胺酶最为常见 ${ }^{[23]}, \beta$-内酰胺酶通 过其作用位点Ser-OH对 $\beta$-内酰胺环上的羧基碳进行亲 核攻击使环打开, 形成由酯键连接的酯酶复合物中间 体, 而后酯键水解使酶再生, 同时释放已失活的 $\beta$-内酰 胺环部分, 从而介导细菌产生耐药性 ${ }^{[24]}$. 用 $\beta$-内酰胺类 药物(氨茮西林、美罗培南、亚胺培南、头狍他啶、 厄他培南、头狍噻肟等)孵育耐药细菌一定时间后, 含 有 $\beta$-内酰胺酶活性的耐药菌会引起抗菌药物的水解, 从而造成+18 Da的质量偏移, 通过MALDI-TOF MS准 确识别该种质量的改变可鉴别细菌的耐药性与敏感 性. Roncarati等人 ${ }^{[25]}$ 通过上述方法测定革兰氏阴性菌 血培养阳性发酵液中头孢噻肟和厄他培南的水解情 况, 从而对超广谱 $\beta$-内酰胺酶(extended spectrum $\beta$ lactamases, ES $\beta \mathrm{L}$ )和碳青霉烯酶进行分析, 结果与全 自动药敏分析系统VITEK2比较后表明, 两种方法一 致性较好. 药敏分析系统需要标准的待测菌悬液, 因 此仍然依赖于微生物的前期培养, 而MALDI-TOF MS 技术直接分析血细胞阳性发酵液, 一定程度上缩短了 时间周期. 由于同种菌群中细菌耐药存在异质性, 发 
展可用于探究细菌群体组成并确定细菌耐药异质性的 单细胞分析方法具备重要的理论与实践意义. Dai等 人 ${ }^{[26]}$ 构建了等离激元微胶囊-MALDI-TOF MS联用平 台, 其中微胶囊可用于封装单个细菌, 用作单细菌催 化下的抗生素水解反应器, 并直接作为MALDI样品点 进行质谱分析, 由此从单细胞水平上对大肠杆菌中 $\beta$ 内酰胺酶介导的抗生素耐药异质性进行研究.

质粒介导产生的氨基糖苷类钝化酶，由革兰氏阴 性菌及部分革兰氏阳性菌产生，主要分为三类：N-乙 酰转移酶、腺苷酸转移酶、磷酸转移酶, 其分别通过 乙酰化、腺苷酸化以及磷酸化对抗生素进行修饰从而 使其失活 ${ }^{[27,28]}$. 以肠杆菌科菌株中由质粒介导产生的 喹诺酮乙酰化耐药机制为例, Pardo 等人 ${ }^{[29]}$ 借助MALDI-TOF MS研究113株肠杆菌科细菌中AAC $\left(6^{\prime}\right)-\mathrm{Ib}-\mathrm{cr}$ 乙酰转移酶对诺氟沙星的活性, 发现在 $\mathrm{AAC}\left(6^{\prime}\right)-\mathrm{Ib}-\mathrm{cr}$ 酶作用下，诺氟沙星及其钠加合物的分子峰强度明显 降低，相比而言，乙酰化诺氟沙星及其钠加合物在相 对分子质量 $+42 \mathrm{Da}$ 处产生明显的检测信号，该方法使 用 $0.03 \mathrm{~g} / \mathrm{L}$ 的药物浓度检测时，结果与Wachino表型实 验的灵敏度和特异性无显著差异.

(2) 稳定同位素(非放射性)标记的氨基酸检测. 细 菌耐药机制多样, 除产生耐药相关酶外, 还可能归因于 抗生素作用靶位改变、细菌膜通透性降低、主动外排 系统过表达等机制 ${ }^{[30]}$. 对于不涉及抗生素降解或修饰 的耐药机制，可对细菌摄入的氨基酸进行同位素标记， 通过观察代谢水平差异实现间接检测，只有具有抗性 的微生物在生长过程中能够被掺入标记的氨基酸，对 应谱图中相应峰的质量转移, 通过检测包含标记的重 氨基酸的抗性分离物可实现敏感株与耐药株的鉴 定 ${ }^{[31]}$. Jung 等人 ${ }^{[32]}$ 选择铜绿假单胞菌作为模型生物, 并 将菌株在正常培养基，含有 ${ }^{13} \mathrm{C}_{6}{ }^{15}{ }^{15} \mathrm{~N}_{2}$ 标记的赖氨酸培 养基，以及同时包括标记赖氨酸与抗生素的培养基中 孵育，成功测试了铜绿假单胞菌分离株对美罗培南、 妥布霉素和环丙沙星的耐药性.

(3) 耐药相关生物标志物的特征性质谱峰检测. 基 于耐药菌株与敏感菌株蛋白质表达差异，以特异性蛋 白质作为生物标志物，依据单峰或峰簇差异可进一步 鉴别敏感株与耐药株 ${ }^{[33]}$. Gaibani等人 ${ }^{[34]}$ 通过ClinProtTools软件(v3.0)分析KPC阳性与KPC阴性肺炎克雷伯 菌蛋白质谱图，经单峰分析篮选出 $11109 \mathrm{Da}$ 谱峰作为 KPC阳性肺炎克雷伯菌的特异性生物标志物。该研究
与一项回顾性研究 ${ }^{[35]}$ 存在相关性. 回顾研究表明, 11109 Da峰对应于肺炎克雷伯菌pKpQIL质粒中p019 假设蛋白的裂解产物，可用于表征携带bla(KPC)碳青 霉烯酶基因的 $\mathrm{pKpQIL}$ 质粒是否存在.

\section{MALDI-TOF MS技术在核酸检测方面 的应用}

鉴于MALDI-TOF MS应用于菌种鉴定时主要依 赖核糖体蛋白的肽质量指纹图谱与数据库中已有信息 的比对, 而病毒无细胞结构不含有核糖体蛋白, 其主要 成分为核酸与含量较少、类型多样的蛋白质，转录复 制依赖于宿主细胞 ${ }^{[36]}$ ，因此基于蛋白质检测的MALDI-TOF MS系统不是病毒鉴定的主流方法. 质谱应用 于核酸检测技术的出现，弥补了其在病毒检测方面的 局限．该技术将PCR与质谱技术联用，可实现包含病 毒与细菌等在内的多种病原体的检测与分型，也适用 于耐药相关基因的检测(图1).

\section{1 病原体检测与分型}

伴随分子诊断领域的快速发展，辅助核酸电离的 基质相继被发现, MALDI-TOF MS技术也被广泛应用 于核酸序列多样性的鉴定工作中，如单核苷酸多态性 (single nucleotide polymorphism, SNP)、拷贝数变 化、基因修饰如DNA甲基化等 ${ }^{[37]}$. 多种质谱平台如 MassARRAY ${ }^{[38]}$, QuanSNP ${ }^{[39]}$ 的MALDI-TOF MS技术 应用兼具了PCR技术的高灵敏度、芯片技术的高通 量以及质谱技术的高准确性，其最大的优势在于多重 反应通量较高，一般单个反应可达10 60重检测，选用 96孔、384孔制式的芯片，可满足不同检测量的需求， 同时检测周期较短，支持不同类型标志物的检测 ${ }^{[40]}$. 以MALDI-TOF MS应用于SNP检测为例，其技术原理 为：首先利用多重PCR技术扩增包含SNP位点在内的 DNA序列；随后针对每个靶点SNP设计一条延伸引 物, 在以ddNTPs为底物的体系中进行单碱基延伸; 最 后利用MALDI-TOF MS平台对延伸产物进行分析, 依 据待测样品的核苷酸多态性对病原体进行检测与分 型 ${ }^{[41]}$ (表1)。目前有多个MALDI-TOF MS平台应用该 原理开展了各类病原体的检测，如性传播病原体、肺 炎支原体、多瘤病毒、肠道病毒、呼吸道病毒、呼 吸道细菌、人冠状病毒、人乳头瘤病毒、疮疹病毒 


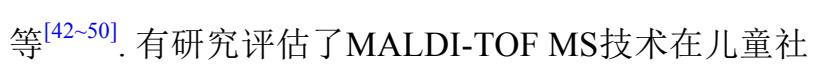
区获得性肺炎诊疗工作中的适用性, 研究选取302例 患儿痰液进行质谱分析, 成功鉴定出 19 种相关病毒与 12 种相关细菌, 在此基础上确立抗生素治疗方案并与 最初的经验疗法进行比较, 结果表明 $79.7 \%$ 的患儿初 始治疗方案有待改进, 提示利用MALDI-TOF MS技 术可快速鉴定病原体并有助于指导抗生素治疗 ${ }^{[51]}$. 赵 飞等人 ${ }^{[52]}$ 借助QuanSNP技术平台成功建立了肺炎支 原体多位点SNP基因分型与大环内酯易感基因检测 方法, 方法特异性达 $100 \%$, 应用于临床样本中检出限 可达 $5.2 \times 10^{2}$ 拷贝/反应, 有助于提高临床肺炎支原体 基因分型能力. 在应对新冠肺炎疫情的过程中, 利用 MALDI-TOF MS平台可发挥多重检测的优势, 基于该 平台开发出的用于SARS-CoV-2检测与分型的方法, 与 $\mathrm{qPCR}$ 进行比对分析, 两者一致性达 $100 \%$, 提示 MALDI-TOF MS技术或可作为常规核酸检测手段的 替代方法, 提高病原检测的时效 ${ }^{[53]}$. 该方法的不足之 处在于需要特定的设备、不能检测未知突变、操作 相对复杂等, 因此在检测条件有限的实验室应用受限, 适用于已具备相关仪器的实验室进行大量样本的多 位点分型检测。

\section{2 细菌耐药性预测}

表型特征改变通常可追溯到基因变化, 耐药性一 般由多因素综合作用产生, 如染色体基因的自发突变, 以及通过质粒、转座子、噬菌体等在种内或种间进行 遗传元件的转移 ${ }^{[54]}$, 由此, 通过检测耐药相关基因可 对菌株耐药性进行预测(表1). 淋病奈瑟菌是常见的性 传播病原菌, 逐步增长的耐药性成为淋病诊疗与防治 的难点. 李雅梅等人 ${ }^{[55]}$ 借助MALDI-TOF MS平台多重 基因检测的优势, 将其应用于淋病奈瑟菌耐药相关位 点的快速检测, 从而预测耐药性. 该方法可同时检测 淋病奈瑟菌9个抗菌药物耐药性相关基因中的 24 个突 变. 通过检测454株已知最低抑菌浓度的淋病奈瑟菌分 离株、8株奈瑟菌属菌株以及214份临床样本来评估方 法的灵敏度与特异度. 结果表明, 针对淋病奈瑟菌耐药 位点的MALDI-TOF MS检测方法, 可在高通量水平上 提供耐药基因的变异信息, 并可直接应用于临床标本 的抗菌药物耐药性篎查, 为全面的流行病学调查与个 性化治疗方案提供依据.

\section{4 总结与展望}

MALDI-TOF MS作为病原微生物研究领域的重 要手段, 被广泛应用于菌种鉴定、耐药性检测、病原 体检测与分型中, 具备高通量、低成本、高准确性等 特点. 相比临床微生物与公共卫生实验室中常规的表 型微生物鉴定手段, MALDI-TOF MS在一定程度上 缩短了鉴定分型时间，简化了操作程序，某些情况下 或可直接从临床标本中快速准确地鉴别微生物, 有助 于为及时制定有效的治疗方案提供依据, 从而改善患 者的预后效果 ${ }^{[51,56]}$. 与常规分子生物学检测方法进行 比较, MALDI-TOF MS能满足高通量检测的要求, 同 时仪器操作简便, 结果具备可重复性与稳定性 ${ }^{[57 ~ 59]}$. 另有研究针对MALDI-TOF MS与全自动微生物鉴定 系统以及传统生化检测试剂盒等方法的准确性、鉴 定时间和成本进行了评估, 量化了其作为一线鉴定方 法时所需时间和成本的优势 ${ }^{[60]}$. MALDI-TOF MS技 术的可靠性和准确性已在许多研究中得到验证, 该方 法在完善菌株鉴定及指导抗生素使用方面具备重大 潜能.

尽管MALDI-TOF MS正作为一项迅速发展的前 沿技术逐步进入到一线微生物鉴定工作中, 但在某些 方面仍然存在技术局限性. 鉴于将待测菌株的PMF与 数据库中存在的参考质量指纹图谱进行比较是菌种鉴 定的关键步骤, 而少数具有相似遗传背景的菌株不易 通过质谱信号区分并获得高度一致的鉴定结果, 因此 不断更新参考数据库对于提高检测性能至关重要. 同 时, MALDI-TOF MS系统中应进一步扩大快速识别抗 生素抗性特征的数据库, 以期在耐药性检测及抗生素 合理应用方面发挥更大功效. 另外, 基质种类和样品 制备将直接影响结果的分析, 因此优化基质的选择或 改进样品的前处理方式, 对于开发从复杂混合物中选 择性检测目标生物分子的MALDI-TOF MS方法具有 重要的实践意义.

已知病原体耐药问题亟待解决, 各类新发突发传 染病尚未可知, 微生物鉴定与分型工作任重道远, MALDI-TOF MS技术处在活跃发展的阶段, 可有效填 补常规检测手段的空缺, 除应用于科学研究领域外, 在 传染病临床诊疗方面也具备极大的开拓空间. 例如, 进 一步开发MALDI-TOF MS多肽指纹图谱分析能力, 通 过比对不同传染性疾病之间的峰图建立检测模型, 扩 
展可鉴定微生物的种类和范围; 优化临床样本前处理 手段或开发不同的技术联用，以逐步扩大MALDITOF MS技术在临床样本直接鉴定方面的应用; 发挥 高特异性、高灵敏度以及多重检测优势以更大范围地
监测耐药性指征等. 同时, 作为微生物研究领域内新兴 发展的技术, 应加强对其临床相关性能的评估, 注重不 同系统的比较分析与多中心综合验证，以期在病原体 鉴定分型与抗生素耐药分析中发挥更大作用.

\section{参考文献}

1 Ferone M, Gowen A, Fanning S, et al. Microbial detection and identification methods: bench top assays to omics approaches. Compr Rev Food Sci Food Saf, 2020, 19: 3106-3129

2 Tran A, Alby K, Kerr A, et al. Cost savings realized by implementation of routine microbiological identification by matrix-assisted laser desorption ionization-time of flight mass spectrometry. J Clin Microbiol, 2015, 53: 2473-2479

3 Oviaño M, Rodríguez-Sánchez B, Gómara M, et al. Direct identification of clinical pathogens from liquid culture media by MALDI-TOF MS analysis. Clin Microbiol Infect, 2017, 24: 624-629

4 Hou T Y, Chiang-Ni C, Teng S H. Current status of MALDI-TOF mass spectrometry in clinical microbiology. J Food Drug Anal, 2019, 27: 404414

5 Nadler W M, Waidelich D, Kerner A, et al. MALDI versus ESI: the impact of the ion source on peptide identification. J Proteome Res, 2017, 16: $1207-1215$

6 Shao W, Lam H. Tandem mass spectral libraries of peptides and their roles in proteomics research. Mass Spectrom Rev, 2017, 36: 634-648

7 Kostrzewa M, Nagy E, Schröttner P, et al. How MALDI-TOF mass spectrometry can aid the diagnosis of hard-to-identify pathogenic bacteriathe rare and the unknown. Expert Rev Mol Diagn, 2019, 19: 667-682

8 Clark A E, Kaleta E J, Arora A, et al. Matrix-assisted laser desorption ionization-time of flight mass spectrometry: a fundamental shift in the routine practice of clinical microbiology. Clin Microbiol Rev, 2013, 26: 547-603

9 Quintilla R, Kolecka A, Casaregola S, et al. MALDI-TOF MS as a tool to identify foodborne yeasts and yeast-like fungi. Int J Food Microbiol, 2018, 266: 109-118

10 Wigmann É F, Behr J, Vogel R F, et al. MALDI-TOF MS fingerprinting for identification and differentiation of species within the Fusarium fujikuroi species complex. Appl Microbiol Biotechnol, 2019, 103: 5323-5337

11 O'Connor J A, Lynch-Healy M, Corcoran D, et al. Improved matrix-assisted laser desorption ionization-time of flight mass spectrometry (MALDI-TOF MS)-based identification of Mycobacterium spp. by use of a novel two-step cell disruption preparatory technique. J Clin Microbiol, 2016, 54: 495-496

12 Tsuchida S, Umemura H, Nakayama T. Current status of matrix-assisted laser desorption/ionization-time-of-flight mass spectrometry (MALDITOF MS) in clinical diagnostic microbiology. Molecules, 2020, 25: 4775

13 Zhou M, Yang Q, Kudinha T, et al. An improved in-house MALDI-TOF MS protocol for direct cost-effective identification of pathogens from blood cultures. Front Microbiol, 2017, 8: 1824

$14 \mathrm{Li} \mathrm{W}$, Sun E, Wang Y, et al. Rapid identification and antimicrobial susceptibility testing for urinary tract pathogens by direct analysis of urine samples using a MALDI-TOF MS-based combined protocol. Front Microbiol, 2019, 10: 1182

15 Segawa S, Sawai S, Murata S, et al. Direct application of MALDI-TOF mass spectrometry to cerebrospinal fluid for rapid pathogen identification in a patient with bacterial meningitis. Clin Chim Acta, 2014, 435: 59-61

16 De Florio L, Riva E, Giona A, et al. MALDI-TOF MS identification and clustering applied to enterobacter species in nosocomial setting. Front Microbiol, 2018, 9: 1885

17 Park J H, Jang Y, Yoon I, et al. Comparison of Autof ms1000 and Bruker Biotyper MALDI-TOF MS platforms for routine identification of clinical microorganisms. Biomed Res Int, 2021, 2021: 1-10

18 Lin J N, Teng S H, Lai C H, et al. Comparison of the Vitek MS and Bruker Matrix-assisted laser desorption ionization-time of flight mass spectrometry systems for identification of chryseobacterium isolates from clinical specimens and report of uncommon chryseobacterium infections in humans. J Clin Microbiol, 2018, 56: e00712-18

19 Wei J, Zhang H, Zhang H, et al. Novel strategy for rapidly and safely distinguishing Bacillus anthracis and Bacillus cereus by use of peptide mass 
fingerprints based on matrix-assisted laser desorption ionization-time of flight mass spectrometry. J Clin Microbiol, 2020, 59

20 Lévesque S, Dufresne P J, Soualhine H, et al. A side by side comparison of Bruker Biotyper and VITEK MS: utility of MALDI-TOF MS technology for microorganism identification in a public health reference laboratory. PLoS ONE, 2015, 10: e0144878

21 Yi Q, Xiao M, Fan X, et al. Evaluation of Autof MS 1000 and Vitek MS MALDI-TOF MS system in identification of closely-related yeasts causing invasive fungal diseases. Front Cell Infect Microbiol, 2021, 11

22 Vrioni G, Tsiamis C, Oikonomidis G, et al. MALDI-TOF mass spectrometry technology for detecting biomarkers of antimicrobial resistance: current achievements and future perspectives. Ann Transl Med, 2018, 6: 240

23 Bassetti M, Poulakou G, Ruppe E, et al. Antimicrobial resistance in the next 30 years, humankind, bugs and drugs: a visionary approach. Intensive Care Med, 2017, 43: 1464-1475

24 Lima L M, Silva B N M, Barbosa G, et al. $\beta$-lactam antibiotics: an overview from a medicinal chemistry perspective. Eur J Med Chem, 2020, 208: 112829

25 Roncarati G, Foschi C, Ambretti S, et al. Rapid identification and detection of $\beta$-lactamase-producing Enterobacteriaceae from positive blood cultures by MALDI-TOF/MS. J Glob Antimicrob Resist, 2021, 24: 270-274

26 Dai Y, Li C, Yi J, et al. Plasmonic colloidosome-coupled MALDI-TOF MS for bacterial heteroresistance study at single-cell level. Anal Chem, 2020, 92: 8051-8057

27 Ahmed S, Sony S A, Chowdhury M B, et al. Retention of antibiotic activity against resistant bacteria harbouring aminoglycoside-Nacetyltransferase enzyme by adjuvants: a combination of in-silico and in-vitro study. Sci Rep, 2020, 10: 19381

28 Dharuman S, Wilt L A, Liu J, et al. Synthesis, antibacterial action, and ribosome inhibition of deoxyspectinomycins. J Antibiot, 2021, 74: 381396

29 Pardo C A, Tan R N, Hennequin C, et al. Rapid detection of AAC(6')-Ib-cr production using a MALDI-TOF MS strategy. Eur J Clin Microbiol Infect Dis, 2016, 35: 2047-2051

30 Peng Z, Jin D, Kim H B, et al. Update on antimicrobial resistance in clostridium difficile: resistance mechanisms and antimicrobial susceptibility testing. J Clin Microbiol, 2017, 55: 1998-2008

31 Sparbier K, Lange C, Jung J, et al. MALDI biotyper-based rapid resistance detection by stable-isotope labeling. J Clin Microbiol, 2013, 51: 37413748

32 Jung J S, Eberl T, Sparbier K, et al. Rapid detection of antibiotic resistance based on mass spectrometry and stable isotopes. Eur J Clin Microbiol Infect Dis, 2014, 33: 949-955

33 Maus A, Bisha B, Fagerquist C, et al. Detection and identification of a protein biomarker in antibiotic-resistant Escherichia coli using intact protein LC offline MALDI-MS and MS/MS. J Appl Microbiol, 2020, 128: 697-709

34 Gaibani P, Galea A, Fagioni M, et al. Evaluation of matrix-assisted laser desorption ionization-time of flight mass spectrometry for identification of KPC-producing Klebsiella pneumoniae. J Clin Microbiol, 2016, 54: 2609-2613

35 Lau A F, Wang H, Weingarten R A, et al. A rapid matrix-assisted laser desorption ionization-time of flight mass spectrometry-based method for single-plasmid tracking in an outbreak of carbapenem-resistant Enterobacteriaceae. J Clin Microbiol, 2014, 52: 2804-2812

36 Napthine S, Bell S, Hill C H, et al. Characterization of the stimulators of protein-directed ribosomal frameshifting in Theiler's murine encephalomyelitis virus. Nucleic Acids Res, 2019, 47: 8207-8223

37 Gao X, Tan B H, Sugrue R J, et al. MALDI mass spectrometry for nucleic acid analysis. In: Cai Z, Liu S, eds. Applications of MALDI-TOF Spectroscopy. Topics in Current Chemistry. Berlin, Heidelberg: Springer, 2013. 55-77

38 Lamy P J, van der Leest P, Lozano N, et al. Mass spectrometry as a highly sensitive method for specific circulating tumor DNA analysis in NSCLC: a comparison study. Cancers, 2020, 12: 3002

39 Liu N, Wang L, Cai G, et al. Establishment of a simultaneous detection method for ten duck viruses using MALDI-TOF mass spectrometry. J Virol Methods, 2019, 273: 113723

40 Huang C H, Li S W, Huang L, et al. Identification and classification for the Lactobacillus casei Group. Front Microbiol, 2018, 9: 1974

41 Syrmis M W, Moser R J, Whiley D M, et al. Comparison of a multiplexed MassARRAY system with real-time allele-specific PCR technology for genotyping of methicillin-resistant Staphylococcus aureus. Clin Microbiol Infect, 2011, 17: 1804-1810

42 Xiu L, Zhang C, Li Y, et al. Simultaneous detection of eleven sexually transmitted agents using multiplexed PCR coupled with MALDI-TOF analysis. IDR, 2019, Volume 12: 2671-2682 
43 Xiao D, Zhao F, Zhang H, et al. Novel strategy for typing Mycoplasma pneumoniae isolates by use of matrix-assisted laser desorption ionizationtime of flight mass spectrometry coupled with ClinProTools. J Clin Microbiol, 2014, 52: 3038-3043

44 Peng J, Li K, Zhang C, et al. MW polyomavirus and STL polyomavirus present in tonsillar tissues from children with chronic tonsillar disease. Clin Microbiol Infect, 2016, 22: 97.e1-97.e3

45 Peng J, Yang F, Xiong Z, et al. Sensitive and rapid detection of viruses associated with hand foot and mouth disease using multiplexed MALDITOF analysis. J Clin Virol, 2013, 56: 170-174

46 Zhang C, Xiao Y, Du J, et al. Application of multiplex PCR coupled with matrix-assisted laser desorption ionization-time of flight analysis for simultaneous detection of 21 common respiratory viruses. J Clin Microbiol, 2015, 53: 2549-2554

47 Zhang C, Xiu L, Xiao Y, et al. Simultaneous detection of key bacterial pathogens related to pneumonia and meningitis using multiplex PCR coupled with mass spectrometry. Front Cell Infect Microbiol, 2018, 8: 107

48 Xiu L, Zhang C, Wu Z, et al. Establishment and application of a universal coronavirus screening method using MALDI-TOF Mass spectrometry. Front Microbiol, 2017, 8: 1510

49 Peng J, Gao L, Guo J, et al. Type-specific detection of 30 oncogenic human papillomaviruses by genotyping both E6 and L1 genes. J Clin Microbiol, 2013, 51: 402-408

50 Xiao H, Liu W, Zhao Z, et al. Single nucleotide polymorphism rs2274084 of gap junction protein beta 2 gene among Epstein-Barr virusassociated tumors. Cancer Biomark, 2018, 21: 499-504

51 Sun L, Zhang C, An S, et al. Comprehensive description of pathogens and antibiotic treatment guidance in children with community-acquired pneumonia using combined mass spectrometry methods. Front Cell Infect Microbiol, 2021, 11: 695134

52 Zhao F, Zhang J, Wang X, et al. A multisite SNP genotyping and macrolide susceptibility gene method for Mycoplasma pneumoniae based on MALDI-TOF MS. iScience, 2021, 24: 102447

53 Wandernoth P, Kriegsmann K, Groh-Mohanu C, et al. Detection of severe acute respiratory syndrome coronavirus 2 (SARS-CoV-2) by mass spectrometry. Viruses, 2020, 12: 849

54 McEwen S A, Collignon P J. Antimicrobial resistance: a one health perspective. Microbiol Spectr, 2018, 6

$55 \mathrm{Li} \mathrm{Y,} \mathrm{Xiu} \mathrm{L,} \mathrm{Liu} \mathrm{J,} \mathrm{et} \mathrm{al.} \mathrm{A} \mathrm{multiplex} \mathrm{assay} \mathrm{for} \mathrm{characterization} \mathrm{of} \mathrm{antimicrobial} \mathrm{resistance} \mathrm{in} \mathrm{Neisseria} \mathrm{gonorrhoeae} \mathrm{using} \mathrm{multi-PCR} \mathrm{coupled}$ with mass spectrometry. J Antimicrob Chemother, 2020, 75: 2817-2825

56 Yuan Y, Wang J, Zhang J, et al. Evaluation of an optimized method to directly identify bacteria from positive blood cultures using MALDI-TOF mass spectrometry. J Clin Lab Anal, 2020, 34

57 van Veen S Q, Claas E C J, Kuijper E J. High-throughput identification of bacteria and yeast by matrix-assisted laser desorption ionization-time of flight mass spectrometry in conventional medical microbiology laboratories. J Clin Microbiol, 2010, 48: 900-907

58 Bizzini A, Durussel C, Bille J, et al. Performance of matrix-assisted laser desorption ionization-time of flight mass spectrometry for identification of bacterial strains routinely isolated in a clinical microbiology laboratory. J Clin Microbiol, 2010, 48: 1549-1554

59 Jussiaux F, Miot-Sertier C, Nguyen-Lopez D, et al. Reliability of MALDI-TOF mass spectrometry to identify oral isolates of Streptococcus salivarius and Lactobacillus spp. Arch Oral Biol, 2021, 121: 104983

60 Tan K E, Ellis B C, Lee R, et al. Prospective evaluation of a matrix-assisted laser desorption ionization-time of flight mass spectrometry system in a hospital clinical microbiology laboratory for identification of bacteria and yeasts: a bench-by-bench study for assessing the impact on time to identification and cost-effectiveness. J Clin Microbiol, 2012, 50: 3301-3308 


\title{
Progress in applications of MALDI-TOF MS technology in the diagnosis of infectious diseases
}

\author{
WANG LiQin $^{1,2}$, ZHANG Chi ${ }^{1,2}$, LI XiaoDong ${ }^{3}$ \& PENG JunPing ${ }^{1,2}$ \\ 1 NHC Key Laboratory of Systems Biology of Pathogens, Institute of Pathogen Biology, Chinese Academy of Medical Sciences \& \\ Peking Union Medical College, Beijing 100176, China; \\ 2 Key Laboratory of Respiratory Disease Pathogenomics, Chinese Academy of Medical Sciences \& Peking Union Medical College, \\ Beijing 100176, China; \\ 3 Shimadzu China Innovation Center, Shimadzu (China) Co. LTD, Beijing 100020, China
}

\begin{abstract}
Matrix-assisted laser desorption/ionization time-of-flight mass spectrometry (MALDI-TOF MS) has been widely used in clinical microbiology laboratories as an emerging detection method. Compared with traditional phenotypic-based detection methods such as morphology, serology, and biochemical detection, MALDI-TOF MS can identify the microorganisms and analysis phenotype of antimicrobial resistance in a short time, simplifying the operation process. A number of studies have shown that MALDI-TOF MS has great application potential in the field of pathogenic biology. It is suitable for the detection of various molecules by analyzing the mass-to-charge ratio of molecules or ions to identify or quantify the analyte. This article systematically reviews the progress in applications of MALDI-TOF MS in the diagnosis of infectious diseases from two aspects of protein and nucleic acid depending on the detection targets.
\end{abstract}

MALDI-TOF MS, bacteria identification, antimicrobial resistance, pathogen detection and typing

doi: $10.1360 / \mathrm{SSV}-2021-0317$ 